\title{
Das Schweizer Lebendspenderregister und die Krankenkassen
}

Gilbert T. Thiel

Korrespondenz:

Prof. Dr. med. Gilbert T. Thiel Fliederweg 5 CH-4103 Bottmingen

gil.thiel@unibas.ch
Das Schweizer Lebendspenderregister startete im April 1993, zu einem Zeitpunkt, als die Nierenlebendspende in der Schweiz rasch zunahm, diese Entwicklung aber mit Besorgnis beobachtet wurde. Sind die Risiken der Lebendspende zumutbar? Nirgendwo gab es prospektiv erhobene Daten. Ein Bedürfnis danach war gross. Das Register erhielt den Namen SOL-DHR (Swiss Organ Living Donor Health Registry). Es war das erste weltweit. Die Ziele von SOL-DHR waren und sind die:

- prospektive und longitudinale Erfassung des Gesundheitszustandes der Nierenlebendspender aus allen sechs Schweizer Transplantationszentren (seit 2008 auch der Leberlebendspender), überprüft in definierten Zeitabständen;

- Beschreibung und Quantifizierung der Frühund Spätkomplikationen (Grundlage zur Information potentieller Spender und Ärzte über die Risiken);

- Veranlassung periodischer Laboruntersuchungen in Blut und Urin, zentral durchgeführt mit gleicher Methodologie;

- Alarmierung des Hausarztes, wenn sich Gefahren abzeichnen und Massnahmen aufdrängen;

- Schaffung einer neutralen Anlaufstelle für Spender mit Problemen, z. B. mit der Krankenkasse oder dem Transplantationszentrum;

- Qualitätskontrolle (bewähren sich neue Techniken im Quervergleich? Wer bedauert später die Spende, wie viele sind es und weshalb? usw.).

\section{Der aktuelle Stand}

Die ärztlichen Kontrollen einer Nierenlebendspende erfolgen im Prinzip vor der Spende und dann alle zwei Jahre nach der Spende zeitlich unbegrenzt. SOL-DHR verschickt zu diesen Zeitpunkten ein kleines Paket an die Spender, das die Fragebogen für den Spender selbst (aktueller sozialer Status, SF-8-Test zum Erfassen des derzeitigen Wohlbefindens) und dessen Arzt (Fragebogen zur Untersuchung, Blut- und Urinröhrchen, vorfrankiertes Versandmaterial) enthält. Der Spender geht damit zu seinem Hausarzt. Die ausgefüllten Fragebögen werden dem SOL-DHR retourniert, die Blut- und Urinröhrchen an das

\section{Le Registre suisse des donneurs \\ vivants et les caisses-maladie}

Depuis 1993, le Registre suisse des donneurs vivants (Swiss Organ Living Donor Health Registry SOL-DHR) a recensé plus de 1116 donneurs de reins vivants dans les six centres spécialisés que compte la Suisse. Le suivi des donneurs se passe dans de bonnes conditions grâce à la collaboration avec les médecins de premier recours. Les risques à long terme d'un don de rein peuvent ainsi être reconnus et traités. La prise en charge des coûts est clairement réglée par la Loi sur la transplantation. L'assurance du receveur (sa caisse-maladie ou l'assurance-invalidité pour un enfant) doit assumer les coûts du suivi des donneurs. Malheureusement, ce système ne fonctionne pas très bien - voire uniquement grâce à un grand travail administratif de la part des médecins de famille et du registre - ou même pas du tout. Les mécanismes à la base du disfonctionnement du système de décomptes individuels sont exposés dans le présent article.

Pour résoudre ces problèmes, une modification de système est proposée sous forme d'un «forfait unique» pour un suivi d'une durée de 35 ans (forfait pour donneurs vivants), à verser au moment du don par la caisse-maladie du receveur (ou par l'AI si celui-ci est mineur) et par son canton de domicile, selon la même clé de répartition que pour le forfait de transplantation. La proposition a été soumise aux caisses-maladie et à la Conférence suisse des directrices et directeurs cantonaux de la santé.

Labor Viollier AG in Basel versandt. Die Laborergebnisse erhalten der Hausarzt und SOL-DHR.

Inzwischen sind 1151 (Stand 10. Januar 2009) Nierenlebendspender und seit Januar 2008 auch Leberlebendspender erfasst. In diesen Jahren wurden über 3900 Konsultationen zum gröss- 
ten Teil von Hausärzten durchgeführt und über 14100 laborchemische Messergebnisse aus Blut und Urin gewonnen. Letztere werden von SOLDHR laufend eingegeben, monatlich besprochen und jährlich ausgewertet. Wenn sich im Einzelverlauf eine Gefahr abzeichnet, erhält der Hausarzt einen individuellen Brief mit einer Empfehlung. Solche erfolgen zurzeit in der Grössenordnung von etwa 30 pro Monat. Das kann ein Hinweis sein auf die neu aufgetretene Mikroalbuminurie mit Behandlungsempfehlung oder ein Vorschlag, ob und wie auf eine beobachtete Nierenfunktionsverschlechterung reagiert werden soll. So wurde z.B. bei einer Nierenspenderin nach anfänglich perfektem Verlauf im 7. Jahr nach Spende eine starke, aber asymptomatische Verschlechterung der Nierenfunktion beobachtet. Der Hausarzt wurde alarmiert und leitete die Abklärung ein. Als Ursache wurde eine Hydronephrose in der belassenen Niere festgestellt (neu aufgetretene Urinabflussstörung am pyeloureteralen Übergang). Nach chirurgischer Beseitigung der Engstelle normalisierte sich die Nierenfunktion wieder. Uns ist ein ähnlicher Fall in einem schwedischen Transplantationszentrum bekannt, bei dem der Funktionsverlust erst im symptomatischen Stadium der hydronephrotischen Schrumpfniere entdeckt wurde. Jene Nierenspenderin ist heute an der Dialyse und wartet auf ein Transplantat. 14 Jahre nach Nierenspende weisen im SOL-DHR 60\% der Spender eine Hypertonie auf und 13\% eine Mikroalbuminurie, was beides bei Einnierigen eine Behandlung dringlich erfordert, sobald be-

\section{Abbildung 1}

Spenden- und Untersuchungsstatistik gemäss Schweizer Lebendspenderregister (SOL-OHR).

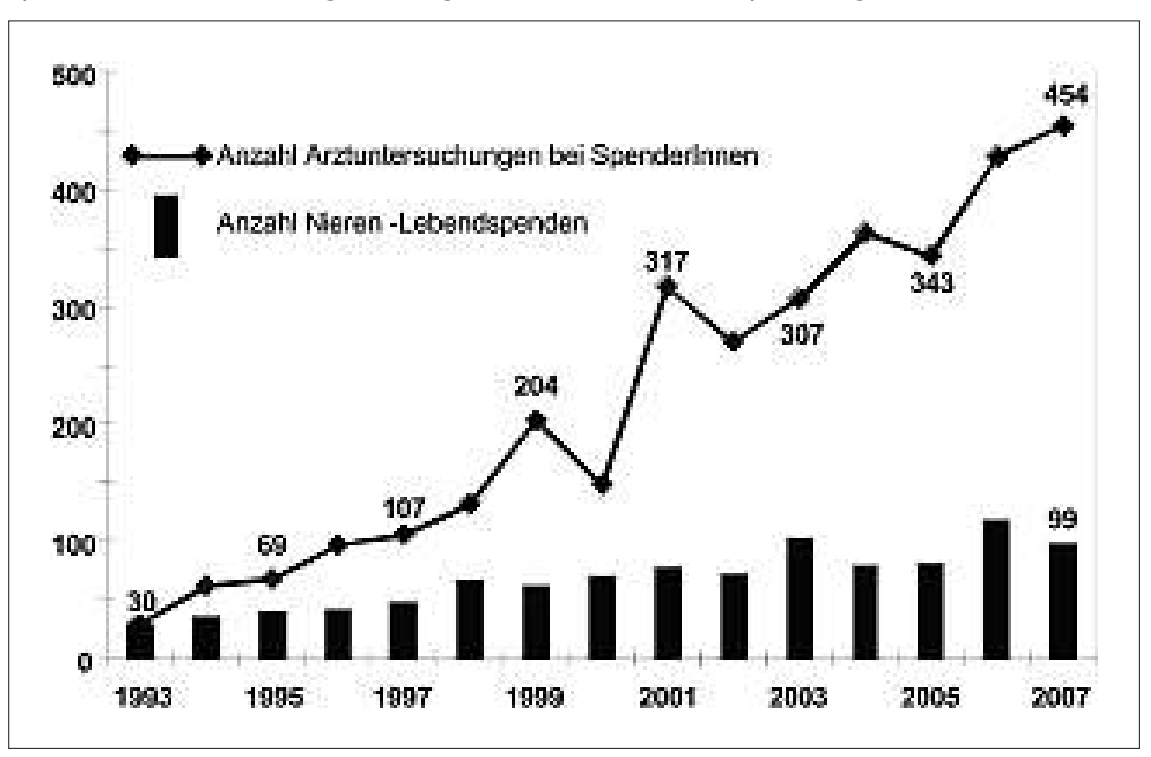

obachtet. Bei einer Spenderin fehlte eine Mikroalbuminurie während 12 Jahren nach Spende und trat erst im 14. Jahr massiv auf. Ein Abbruch der Kontrollen nach 5 Jahren, wenn die Ergebnisse unauffällig sind (ein früherer Vorschlag einer Krankenkasse), vergisst, dass der Stress der Hyperfiltration nach Nephrektomie lebenslang andauert. Es ist so, als wollte man einer Lokomotive, die täglich die Gotthardstrecke zu befahren hat, weitere Maschinenkontrollen ersparen, sobald sie die ersten 5 Jahre tadellos funktioniert hat.

SOL-DHR funktioniert im Auftrag der sechs Transplantationszentren und zur Zufriedenheit der Organspender. Essentiell dabei ist die Einbindung der Hausärzte. Sie gewährleisten eine landesweit einheitliche Betreuung nach Organspende ohne dem Spender die oft weite Reiserei zu einem Transplantationszentrum zumuten zu müssen.

Die seit 1993 freiwillig übernommene Aufgabe der periodischen Spendernachbetreuung ist seit Juli 2007 vom Schweizer Transplantationsgesetz zur Pflicht erklärt worden. Der Kostenträger für die Nachbetreuung ist im Gesetz klar definiert. Es ist der Krankenversicherer des Organempfängers (Krankenkasse oder bei Kindern die IV). Zu den Kosten gehören die Arztuntersuchungen in zweijährlichem Abstand, die chemischen Untersuchungen im zentralen Labor und die Betriebskosten von SOL-DHR, die die periodischen Untersuchungen zeitgerecht auslösen und auswerten.

Seit 1993 wurden die Laboruntersuchungen für Nierenspender von der Viollier AG in Basel kostenlos durchgeführt. Die Viollier AG hat eben beschlossen, die Laboruntersuchungen für SOLDHR auch weiterhin kostenlos anzubieten und das Angebot auch auf Leberlebendspender auszuweiten. Die Betriebskosten von SOL-DHR wurden bisher ausschliesslich von Sponsoren sowohl der Pharmaindustrie (Amgen, Astellas, Baxter, Fresenius, Genzyme, Novartis, Roche, Sanofi, Spirig und Wyeth) als auch von Institutionen (Bundesamt für Gesundheit, Schweizerischer Gesellschaft für Nephrologie; Schweizer Nierenliga, Gabriela Gianoli PR) und privaten Gönnern (Alfred und Erika Bär-Spycher Stiftung, Ehepaar Hans U. und Doris Böhi-Altwegg) getragen.

In der Schweiz hat die Zahl Nierenlebendspenden in den letzten 5 Jahren ein hohes Plateau um etwa 100 erreicht (Säulen in Abb. 1). Hingegen steigt die Zahl ärztlicher Untersuchungen weiter an, was sich aus der kumulativen Natur des Auftrages einer Langzeitnachbetreuung unausweichlich ergibt (Linie in Abb. 1). 


\section{Gute Zusammenarbeit, aber auch Ärger bei Hausärzten - weshalb?}

Wichtig war von allem Anfang an der Einbezug der Hausärzte in die Spendernachbetreuung. Dabei war initial unklar, ob ein Spenderregister mit einer grossen Zahl Hausärzte in allen Schweizer Sprachregionen überhaupt aufbaubar sei. Das ging erfreulicherweise sehr gut. Es bestanden auch Befürchtungen, dass die brieflichen Interventionen bei Hausärzten Ärger hervorrufen bzw. als belehrende Einmischung empfunden werden könnten. Ebenso erfreulicherweise war dies nicht der Fall. Hausärzte betreuen in der Regel kaum mehr als 1-2 Organlebendspender, haben deshalb nichts dagegen, über SOL-DHR einen Zugang zu einem grossen Erfahrungsgut zu erhalten. Essentiell für die Zusammenarbeit ist selbstverständlich, dass die Empfehlungen an die Hausärzte gehen.

Trotz guter Zusammenarbeit gab und gibt es auch Ärger bei Hausärzten über die Spendernachbetreuung im Auftrag von SOL-DHR. Weshalb? Es sind jene Rechnungen für Spenderuntersuchungen, die von einigen Empfängerkrankenkassen trotz gesetzlicher Verpflichtung oft erst nach wiederholten und ultimativen Mahnungen bezahlt werden. Häufig braucht es multiple Telefonate, Faxe, Briefe von SOL-DHR an die Kasse, bis die Zahlung erfolgt. Auch die IV (zuständig für den Organersatz bei erkrankten Kindern) hat sich bisher geweigert, die Kosten für die Spendernachbetreuung (meistens für einen Elternteil) zu übernehmen.

Die stark verzögerte oder ganz ausbleibende Bezahlung von Arztrechnungen löst grosse Verärgerung bei den betroffenen Kollegen und Frust beim SOL-DHR-Team aus. Der Zeitaufwand für SOL-DHR-Mitarbeiter/innen, um einige Kassen schliesslich zur Zahlung zu bewegen, ist riesig und wächst kumulativ mit der Zahl zu betreuender Spender. Die dabei entstehenden Kosten stehen in keiner Proportion zur Höhe der geforderten einzelnen Arzthonorare (70-160 Franken). Im Jahr 2007 mussten ${ }^{2} / 3$ der Gesamteinnahmen von SOL-DHR aus Spenden für Inkassotätigkeit ausgegeben werden. Wie kommt es zu diesem Missstand trotz eindeutiger gesetzlicher Bestimmung? Die Mechanismen dafür sind vielfältig.

\section{Gründe, Mechanismen und Ausreden}

Hauptgrund für den Missstand ist, dass die untersuchte Person (Organspender), auf den die Rechnung lautet, nicht identisch ist mit der versicherten Person (Organempfänger). Die Empfängerkrankenkassen erhalten deshalb Arztrechnungen für die Untersuchung von Personen (Organspender), die oft bei ihnen gar nicht versichert sind.
Solche Rechnungen gehen «direkt in den Reisswolf», wie meine Mitarbeiterin kürzlich von einem Kassenfunktionär erfuhr. Da nützen keine erläuternden Begleitbriefe.

Dass der Reisswolf zugepackt hat, erfährt SOLDHR meist erst, wenn die Mahnungen des Hausarztes bei SOL-DHR eintreffen. Einige Kassen argumentieren dann, dass SOL-DHR (nicht sie) der Garant sei, weil die Rechnungen an SOL-DHR adressiert seien, statt an die Organempfängerkrankenkasse. Hausärzte benutzten in der Tat häufig SOL-DHR als Rechnungsadressaten, weil das Herausfinden der Organempfängerkrankenkasse schwierig ist und das Aufgebot zur Organspenderuntersuchung von SOL-DHR ausging. Der Organspender sollte seinem Hausarzt anlässlich der Konsultation den Namen des Empfängers, dessen aktuelle Krankenkasse samt Mitgliedsnummer mitbringen. Das ist viel verlangt - zuviel, um gut zu funktionieren. Es läuft gegen den normalen Weg der Rechnungsstellung für eine Konsultation.

Bezahlt die Krankenkasse den Betrag korrekt, aber lautend auf den Namen des versicherten Organempfängers, erkennen Hausärzte bisweilen die Zahlung nicht, weil die Honorarforderung abgelegt ist unter dem Namen des Organspenders. Der Arzt mahnt dann SOL-DHR und dieses braucht viel Zeit zum Rekonstruieren und Beheben des Missverständnisses.

Ein anderer Grund ist der ungemeldete Krankenkassenwechsel des Empfängers nach Transplantation. Weder der Organspender, noch der Hausarzt, noch SOL-DHR kennen die neue Krankenkasse des Organempfängers. Die Rechnung geht an die alte Krankenkasse und von dort in den Reisswolf oder an SOL-DHR zurück. Für SOL-DHR beginnt dann die Suche nach der neuen Organempfängerkrankenkasse, was aufwendig ist, weil SOL-DHR keine aktualisierte Adressliste des Organempfängers oder von dessen neuer Kasse führt.

Aussichtslos wird die Bezahlung durch die Krankenkasse, wenn der Organempfänger gestorben ist. Die Lebenserwartung von Organspendern ist wesentlich höher als jene der Organempfänger, weshalb diese Konstellation zunimmt. Der Anspruch von Organspendern auf Nachbetreuung endet selbstverständlich nicht mit dem Tod des Organempfängers.

Ebenso wenig Chance auf reguläre Übernahme hat die Rechnung bisher für eine Spendernachkontrolle, wenn der Organempfänger ein Kind ist und deshalb die IV bezahlen müsste. Die meisten IV-Regionalstellen weigern sich trotz klarer gesetzlicher Grundlage, diese Arztrechnungen zu bezahlen. Am Schluss wurden diese Rechnungen bislang aus Mangel an Besserem aus dem 
SOL-DHR-Spendenpool bezahlt. Dieser Pool ist jährlich neu zu generieren und wird ständig kleiner, während die Zahl der Kinder und Jugendlicher, die ein lebendgespendetes Organ transplantiert erhalten, samt ihren Organspendern kontinuierlich wächst.

Es gibt verschiedene weitere Varianten, wie es zu unbezahlten Arztrechnungen im Rahmen der Lebendspendernachbetreuung kommt. Sie können hier nicht alle aufgezählt werden. Ein besonderer Dank sei statt dessen an jene Krankenkassen gerichtet, die die Spendernachbetreuung anstandslos übernehmen und darauf verzichten, formale Gründe für die Rückweisung oder andere Ausreden anzuführen.

\section{Die Problemlösung}

Als wirksame Problemlösung schlägt SOL-DHR vor, statt Einzelabrechnungen die Zahlung einer einmaligen «Lebendspendepauschale» anlässlich einer Organspende durch die Organempfängerkrankenkasse einzuführen. Es ist dies der letzte Zeitpunkt, an dem die Empfängerkasse am Nierenlebendspender noch interessiert ist.

Die Pauschale in der Grössenordnung von 10000 Franken würde die Spendernachbetreuung für 35 Jahre abdecken. Die Zahl 35 berechnet sich aus dem mittleren Alter der Nierenlebendspender in der Schweiz (50 Jahre) und der Lebenserwartung von 50jährigen (weitere 35 Jahre). In diesem Betrag ist eine mittlere jährliche Teue- rungsrate von $0,8 \%$ mitgerechnet. 10000 Franken entsprechen den Dialysekosten von weniger als drei Monaten, die durch die Lebendspende wegfallen. Die Pauschale würde nach dem gleichen Schlüssel wie die Transplantationspauschale von der Nierenempfängerkrankenkasse und dem Nierenempfängerwohnkanton zu Gunsten des Nierenlebendspenders übernommen. Aus diesem Betrag würde SOL-DHR die zweijährlichen Hausarztrechnungen begleichen, sobald jeweils die Ergebnisse der Untersuchungen eingetroffen sind, sowie die Rechnungen des zentralen Labors und die eigenen Betriebskosten - alles streng kontrolliert. Alle administrativen Schwierigkeiten samt jenen, verknüpft mit dem Krankenkassenwechsel oder Tod des Organempfängers nach Transplantation, wären mit einem Schlag behoben.

Letztlich geht es um eine Grundsatzfrage. Will man den Organlebendspendern eine lebenslange Überwachung jener Langzeitrisiken gewähren, die mit der Organspende zusammenhängen, die man erkennen und behandeln könnte? Das geltende Gesetz und die Richtlinien der SAMW wollen es so. Wenn man es will, muss das gegenwärtige System der Einzelabrechnungen samt beschriebenen administrativen Wirrnissen, Leerläufen und Fallen ersetzt werden durch ein einfaches Pauschalsystem. SOL-DHR droht sonst zu ersticken. Die Entscheidung muss bald erfolgen. Der Vorschlag liegt auf dem Tisch der Krankenkassen und der Gesundheitsdirektorenkonferenz. 